\title{
UMA PROPOSTA DE ALOCAÇÃO DE CONTROLE BASEADA EM MODELO DE REFERÊNCIA DO TIPO PI
}

\author{
${ }^{1}$ N.G. SOUSA e ${ }^{2}$ L.C. OLIVEIRA-LOPES \\ ${ }^{1}$ Universidade Federal do Triângulo Mineiro, Departamento de Engenharia Química \\ ${ }^{2}$ Universidade Federal de Uberlândia, Faculdade de Engenharia Química \\ E-mail para contato: nadia@icte.uftm.edu.br, lcol@ufu.br
}

\begin{abstract}
RESUMO - Este trabalho introduz uma metodologia de controle de alocação baseado em modelo de referência PI (Proporcional-Integral). O controle proposto possibilita a implementação de uma estratégia tolerante a falhas com redundância de atuadores e é capaz de redistribuir o esforço total do controle entre atuadores, mesmo em ambiente com restrição nos atuadores e durante falhas. A vantagem da alocação com modelo de referência é a sintonia intuitiva e a possibilidade de projetar o comportamento desejado para a variável controlada. Para ilustrar e avaliar o controlador proposto, estuda-se o desempenho de controlador para um reator bioquímico com falhas. Os resultados obtidos são promissores quando o espaço de entradas é projetado para os diversos cenários de falhas de interesse.
\end{abstract}

\section{INTRODUÇÃO}

O controle de processos tem sofrido mudanças significativas na tecnologia de instrumentação e controle. Pressões associadas com o aumento da competividade, mudanças rápidas nas condições econômicas, leis ambientais mais rigorosas faz com que controle de processos seja continuamente modernizado nas plantas químicas. Assim, estratégias de controle mais sofisticadas são facilmente justificáveis para manter a operação da planta próxima do ótimo econômico e dentro do limite de segurança, evitando assim, desperdícios de matéria-prima, acidentes e aumentando os lucros. Para um problema de controle, o efeito desejado pode ser obtido por um conjunto de diferentes atuadores. Embora o mais usual na indústria seja o problema de controle para o qual o número de variáveis que se deseja controlar seja superior aquele de variáveis manipuladas disponíveis, o problema com número de variáveis manipuladas superior ao de variáveis controladas ocorre nos casos em que a redundância de atuadores permite ampliar a confiabilidade do sistema.

Uma planta química não está livre de problemas relacionados aos seus equipamentos, nem aos atuadores. Assim, os sistemas industriais exigem estratégias de controle que garanta a tolerância do processo a eventos de falha. Esse desafio tem motivado o estudo de uma estratégia amplamente conhecida na literatura - a alocação de controle. O problema de alocação de controle surge quando o sistema a ser controlado tem mais atuadores físicos do que objetivos de controle. Assim, os atuadores disponíveis precisam ser alocados de alguma forma a atingir os objetivos desejados para o sistema (Basson, 2011). A sua relevância dá-se na utilização dos atuadores em todos os instantes, na utilização de atuadores distintos a depender dos cenários de utilização da 
planta e na necessária mitigação dos efeitos de falhas nos momentos necessários, sem a complexidade de estruturas de controle preditivo baseado em modelo.

A técnica de alocação pode ser encontrada em várias aplicações práticas como a estudada por Zaccarian (2009) que abordou seu estudo em um sistema de controle com redundância de atuadores e propôs um aumento na dinâmica do projeto de controle que realiza a alocação das entradas da planta com o objetivo de empregar cada atuador de forma mais adequada. $\mathrm{O}$ trabalho de Vermillion et al. (2011) estudou a aplicação da alocação com controle preditivo (MPCA) a um sistema de geração de energia térmica utilizando dois atuadores com dinâmicas diferentes. Sistemas que necessitam elevado confiabilidade são os alvos preferenciais dessa tecnologia. Assim, podem ser encontrados estudos que utilizam a alocação de controle aplicada a modelos da aviação civil, como Wendong e Honglun (2013) e Hamayun et. al (2013). Nos trabalhos de Bodson (2002) e Bodson e Frost (2011) propõem-se o uso de modelos de referência representando a planta nominal.

Neste trabalho introduz-se uma metodologia de controle de alocação baseado em modelo de referência PI (Proporcional-Integral). Nessa abordagem não se força o controle a perseguir a dinâmica de uma planta sem falhas, de outra maneira a referência é um processo projetado para ter características desejadas. A vantagem da alocação com modelo de referência PI é a sintonia intuitiva e a possibilidade de projetar o comportamento desejado para a variável controlada. Para ilustrar e avaliar o controlador proposto estuda-se o desempenho do controlador para um reator bioquímico na presença de falhas nos atuadores.

\section{ALOCAÇÃO DE CONTROLE BASEADO EM MODELO DE REFERÊNCIA}

O problema de alocação de controle surge quando o sistema a ser controlado tem mais atuadores físicos que objetivos de controle, ou seja, o número de variáveis manipuladas deve ser maior que o número de variáveis controladas. Os atuadores disponíveis são alocados com a finalidade de atingir os objetivos de controle. Assim, o esforço total do controle é distribuído entre os múltiplos atuadores, essa redistribuição ocorre principalmente durante as falhas. A configuração do controle para a técnica de alocação baseada em modelo de referência é mostrada na Figura 1.

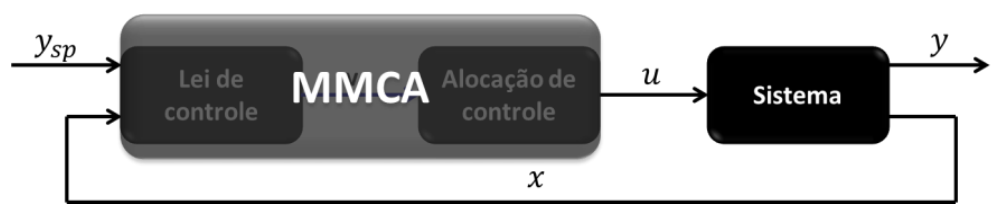

Figura 1 - Configuração do controle para a técnica de alocação.

A MMCA (Model Matching Control Allocation) combina a técnica de alocação com a lei de controle, isso possibilita o uso de controladores clássicos, tipo PI (Proporcional-Integral) e PID (Proporcional-Integral-Derivativo), em um cenário com redundância de atuadores implementados de forma ótima e adicionando-se restrições.

Considere o modelo em espaço de estados representado pela Equação 1: 


$$
\begin{aligned}
& \dot{x}=f(x, u) \\
& y=h(x)
\end{aligned}
$$

em que: $\boldsymbol{x} \in \mathbb{R}^{n}$ é o vetor dos estados, $\boldsymbol{u} \in \mathbb{R}^{m}$ é o vetor de entradas e $\boldsymbol{y} \in \mathbb{R}^{q}$ é o vetor das saídas medidas. O modelo de referência PI é baseado na dinâmica desejada para o sistema em malha fechada, mostrado na Equação 2 .

$$
\frac{d \boldsymbol{y}_{\boldsymbol{r}}}{d t}=\boldsymbol{K}_{c}\left(\boldsymbol{y}_{\boldsymbol{s p}}-\boldsymbol{y}_{\boldsymbol{r}}\right)+\frac{\boldsymbol{K}_{c}}{\boldsymbol{\tau}_{\boldsymbol{I}}} \int\left(\boldsymbol{y}_{s \boldsymbol{p}}-\boldsymbol{y}_{\boldsymbol{r}}\right) d t
$$

em que $y_{\boldsymbol{r}}$ é a saída desejada para o sistema, $\boldsymbol{y}_{\boldsymbol{s} \boldsymbol{p}}$ é o valor do setpoint para as variáveis controladas e $\boldsymbol{K}_{c}$ e $\boldsymbol{\tau}_{I}$ são os parâmetros dos controladores. Escrevendo o modelo de referência em uma representação de espaço de estados tem-se a Equação 3 para cada variável controlada.

$$
\begin{aligned}
& \dot{\boldsymbol{x}_{\boldsymbol{r}}}=\boldsymbol{A}_{\boldsymbol{r}} \boldsymbol{x}_{\boldsymbol{r}}+\boldsymbol{B}_{\boldsymbol{r}} y_{s p} ; \boldsymbol{A}_{\boldsymbol{r}}=\left[\begin{array}{cc}
0 & 2 \\
-\frac{K_{c}}{\tau_{I}} & -K_{c}
\end{array}\right] ; \text { e } \boldsymbol{B}_{\boldsymbol{r}}=\left[\begin{array}{c}
0 \\
\frac{K_{c}}{\tau_{I}}
\end{array}\right] \\
& y_{r}=\boldsymbol{C}_{\boldsymbol{r}} \boldsymbol{x}_{\boldsymbol{r}} ; \boldsymbol{C}_{\boldsymbol{r}}=\left[\begin{array}{ll}
1 & 0
\end{array}\right]
\end{aligned}
$$

No domínio de Laplace cada modelo de referência PI é dado por: $G_{i i}(\mathrm{~s})=\left(2 \tau_{\mathrm{i}} \zeta_{\mathrm{i}} \mathrm{s}+\right.$ 1)/( $\left.\tau_{i}^{2} s^{2}+2 \tau_{i} \zeta_{i} s+1\right)$. O problema de alocação é escrito conforme mostrado na Equação 4.

$$
\begin{aligned}
& \min \left\{\dot{\boldsymbol{y}}-\dot{\boldsymbol{y}}_{\boldsymbol{r}}\right\} \\
& \text { Sujeito a: } \\
& \qquad \begin{array}{c}
\boldsymbol{u}_{\min } \leq \boldsymbol{u} \leq \boldsymbol{u}_{\mathrm{o}} \text { es } \\
\Delta \boldsymbol{u} \leq \Delta \boldsymbol{u}_{\max }
\end{array}
\end{aligned}
$$

Essa formulação de referência PI é similar àquela usada em problemas de controle preditivo baseado em modelos de referência (Kalra et al., 2002). Deseja-se avaliar o valor de $\boldsymbol{u}$ que minimize $\boldsymbol{e}$ (entre outros objetivos) da Equação 5:

$$
e=C_{r} A_{r} x_{r}+C_{r} B_{r} y_{s p}-\frac{d h(x)}{d x} f(x, u)
$$

Nesse trabalho, ilustra-se a alocação da técnica para controladores aplicados a sistemas lineares de ordem relativa unitária e invariantes no tempo, em que $\boldsymbol{a}_{\boldsymbol{d}}$ representa o vetor desejado correspondente a $\boldsymbol{C B u}$,

$$
C B u=-C A+C_{r} A_{r} x_{r}+C_{r} B_{r} y_{s p} \triangleq a_{d}
$$

em que: $\boldsymbol{A}$ é a matriz de estados, $\boldsymbol{B}$ é a matriz de entradas e $\boldsymbol{C}$ é a matriz de saídas. O problema é investigado com duas representações para o problema de otimização:

Problema de alocação com norma $l_{1}$ : O problema de alocação de controle com a norma $l_{1}$ é formulado pela função objetivo mostrada na Equação 7.

$$
\begin{aligned}
& \min J=\left\|\boldsymbol{Q}\left(\boldsymbol{C B} \boldsymbol{u}-\boldsymbol{a}_{\boldsymbol{d}}\right)\right\|_{1}+\left\|\boldsymbol{R}\left(\boldsymbol{u}-\boldsymbol{u}_{\boldsymbol{d}}\right)\right\|_{1}+\|\boldsymbol{S} \Delta \boldsymbol{u}\|_{1} \\
& \text { Sujeito } a: \boldsymbol{u}_{\min } \leq \boldsymbol{u} \leq \boldsymbol{u}_{\text {max }}
\end{aligned}
$$




$$
|\Delta \boldsymbol{u}| \leq \Delta \boldsymbol{u}_{\max }
$$

em que: $\boldsymbol{C B} \boldsymbol{u}-\boldsymbol{a}_{\boldsymbol{d}}=\boldsymbol{e} ; \boldsymbol{u}-\boldsymbol{u}_{\boldsymbol{d}}=\boldsymbol{u}^{+}-\boldsymbol{u}^{-}, \Delta \boldsymbol{u}=\boldsymbol{u}-\boldsymbol{u}_{k-1}, \boldsymbol{e}=\boldsymbol{e}^{+}-\boldsymbol{e}^{-}$, e $\Delta \boldsymbol{u}=\boldsymbol{d}^{+}-\boldsymbol{d}^{-}$. Assim, a alocação é representada pelo problema do tipo LP (Linear Programming Problem) com função objetivo descrito na Equação 8.

$$
J=\sum_{j=1}^{q} \boldsymbol{Q}_{j} \boldsymbol{e}_{j}^{+}+\sum_{j=1}^{q} \boldsymbol{Q}_{\boldsymbol{j}} \boldsymbol{e}_{\boldsymbol{j}}^{-}+\sum_{j=1}^{m} \boldsymbol{R}_{\boldsymbol{j}} \boldsymbol{u}_{\boldsymbol{j}}^{+}+\sum_{j=1}^{m} \boldsymbol{R}_{\boldsymbol{j}} \boldsymbol{u}_{\boldsymbol{j}}^{-}+\sum_{j=1}^{m} \boldsymbol{S}_{\boldsymbol{j}} \boldsymbol{d}^{+}+\sum_{j=1}^{m} \boldsymbol{S}_{\boldsymbol{j}} \boldsymbol{d}^{-}
$$

Problema de alocação com norma $l_{2}$ : O problema de alocação de controle com a norma $l_{2}$ é formulado como um problema do tipo QP (Quadratic Programming Problem) com função objetivo mostrada na Equação 9.

$$
\min J=\left\|\boldsymbol{C} \boldsymbol{B} \boldsymbol{u}-\boldsymbol{a}_{\boldsymbol{d}}\right\|_{Q}^{2}+\left\|\boldsymbol{u}-\boldsymbol{u}_{\boldsymbol{d}}\right\|_{R}^{2}+\|\Delta \boldsymbol{u}\|_{S}^{2}=\frac{1}{2} \boldsymbol{u}^{T} \boldsymbol{Q}_{\boldsymbol{p}} \boldsymbol{u}+\boldsymbol{p}^{T} \boldsymbol{u}
$$

Sujeito a: $\boldsymbol{u}_{\min } \leq \boldsymbol{u} \leq \boldsymbol{u}_{\max }$

$$
|\Delta \boldsymbol{u}| \leq \Delta \boldsymbol{u}_{\max }
$$

em que: $\boldsymbol{Q}_{\boldsymbol{p}}=2\left[\boldsymbol{B}^{T} \boldsymbol{C}^{T} \boldsymbol{Q} \boldsymbol{C} \boldsymbol{B}+\boldsymbol{R}+\boldsymbol{S}\right] ; \boldsymbol{p}^{T}=\left[-2\left(\boldsymbol{a}_{\boldsymbol{d}}{ }^{T} \boldsymbol{Q}^{T} \boldsymbol{C} \boldsymbol{B}+\boldsymbol{u}_{\boldsymbol{d}}{ }^{T} \boldsymbol{R}+\boldsymbol{u}_{\boldsymbol{k}-\mathbf{1}}{ }^{\boldsymbol{T}} \boldsymbol{S}\right)\right]$ e $\boldsymbol{Q}, \boldsymbol{R}$ e $\boldsymbol{S}$ são as matrizes de ponderação do erro em relação à trajetória de referência, em relação à manipulação desejada e na velocidade de variação da variável manipulada, respectivamente.

\section{ESTUDO DO REATOR BIOQUÍMICO}

O sistema estudado consiste em um reator bioquímico exotérmico mostrado na Figura 2.

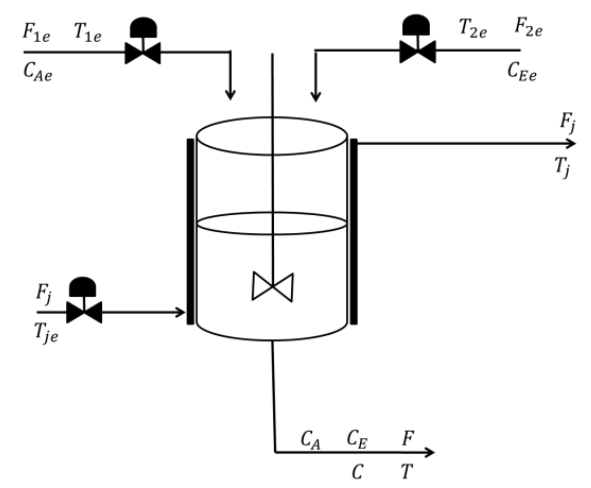

Figura 2 - Esquema do sistema de estudo - reator bioquímico.

em que $C_{A}$ é a concentração do substrato, $C_{E}$ é a concentração da enzima e $C$ é a concentração do produto. O objetivo do sistema é controlar o nível do reator - $h$ e a concentração de produto $-C$, manipulando $F_{1 e}, F_{2 e}$ e $F$. O modelo desse processo é descrito pelas Equações $10-15$.

$$
\frac{d h}{d t}=\frac{F_{1 e}+F_{2 e}-F}{A_{r}}
$$




$$
\begin{aligned}
& \frac{d C_{A}}{d t}=\frac{F_{1 e}}{h A_{r}}\left(C_{A e}-C_{A}\right)-\frac{F_{2 e}}{h A_{r}} C_{A}-\frac{k_{0} e^{-\frac{E}{R T}} C_{A} C_{E}^{2}}{k_{m}+C_{A}} \\
& \frac{d C_{E}}{d t}=\frac{F_{2 e}}{h A_{r}}\left(C_{E e}-C_{E}\right)-\frac{F_{1 e}}{h A_{r}} C_{E} \\
& \frac{d C}{d t}=-\frac{\left(F_{1 e}+F_{2 e}\right)}{h A_{r}} C+\frac{Y_{P / A} k_{0} e^{-\frac{E}{R T}} C_{A} C_{E}^{2}}{k_{m}+C_{A}} \\
& \frac{d T}{d t}=\frac{F_{1 e}}{h A_{r}}\left(T_{1 e}-T\right)+\frac{F_{2 e}}{h A_{r}}\left(T_{2 e}-T\right)-\frac{h A}{\rho c_{p} h A_{r}}\left(T-T_{j}\right)+\frac{\Delta H_{r} k_{0} e^{-\frac{E}{R T} C_{A} C_{E}^{2}}}{\rho c_{p}\left(k_{m}+C_{A}\right)} \\
& \frac{d T_{j}}{d t}=\frac{F_{j}}{V_{j}}\left(T_{j e}-T_{j}\right)+\frac{h A}{\rho_{j} c_{p j} V_{j}}\left(T-T_{j}\right)
\end{aligned}
$$

\begin{tabular}{|c|c|c|}
\hline Parâmetro & Descrição & Valor \\
\hline$F_{1 e} ; F_{2 e} ; F_{j}$ & Vazões de entrada & $2 ; 1 ; 0,2 \mathrm{~m}^{3} / \mathrm{h}$ \\
\hline$F$ & Vazão de saída & $3 \mathrm{~m}^{3} / \mathrm{h}$ \\
\hline$C_{A e} ; C_{E e}$ & Concentrações de entrada & $10 ; 20 \mathrm{~mol} / \mathrm{m}^{3}$ \\
\hline$T_{1 e} ; T_{2 e} ; T_{j e}$ & Temperaturas de entrada & $25 ; 25 ; 15^{\circ} \mathrm{C}$ \\
\hline$A_{r}$ & Área do reator & $5 \mathrm{~m}^{2}$ \\
\hline$V_{j}$ & Volume da jaqueta de refrigeração & $0,71 \mathrm{~m}^{3}$ \\
\hline$\rho ; \rho_{j}$ & Densidade & $960 ; 1000 \mathrm{~kJ} / \mathrm{kg}^{\circ} \mathrm{C}$ \\
\hline$c_{p} ; c_{p j}$ & Capacidade calorífera & 4,$2 ; 4,182 \mathrm{~kJ} / \mathrm{kg}^{0} \mathrm{C}$ \\
\hline$R$ & Constante dos gases & $8,314 \mathrm{~J} / \mathrm{mol} \mathrm{K}$ \\
\hline $\boldsymbol{A}$ & Área de troca térmica & $13,55 \mathrm{~m}^{2}$ \\
\hline$\Delta \boldsymbol{H}_{r}$ & Entalpia da reação & $10000 \mathrm{~kJ} / \mathrm{m}^{2} \mathrm{~h}^{\circ} \mathrm{C}$ \\
\hline $\boldsymbol{E}$ & Energia de ativação & $27,245 \mathrm{~kJ} / \mathrm{mol}$ \\
\hline $\boldsymbol{k}_{0}$ & Constante pré-exponencial & $1,65 * 10^{6} u c$ \\
\hline $\boldsymbol{k}_{m}$ & Constante da reação & $6,8 \mathrm{~mol} / \mathrm{m}^{3}$ \\
\hline $\boldsymbol{h}$ & Coeficiente de transferência térmica & $7200 \mathrm{~kJ} / \mathrm{m}^{2} \mathrm{~h}^{\circ} \mathrm{C}$ \\
\hline$Y_{P / A}$ & Rendimento & 0,5 \\
\hline$\tau_{i} ; \zeta_{i}$ & Parâmetros da referência PI & $20 ; 20 \mathrm{uc}$ \\
\hline$h_{s p 1} ; h_{s p 2}$ & Nível do reator no setpoint 1 e 2 & 2,$25 ; 2,75 m$ \\
\hline$C_{s p 1} ; C_{s p 2}$ & Concentração de produto no setpoint 1 e 2 & 3,$33 ; 3,48 \mathrm{~mol} / \mathrm{m}^{3}$ \\
\hline$Q ; R ; S$ & Matrizes de ponderação & $100 ; 1 ; 1 \mathrm{uc}$ \\
\hline
\end{tabular}

Os parâmetros usados nas simulações estão dispostos na Tabela 1.

Tabela 1 - Parâmetros do sistema

uc: unidades compatíveis

Neste caso, deseja-se que a velocidade do processo se comporte de forma similar ao modelo de referência, que são iguais para cada variável controlada. Ambos os problemas de otimização 
(LP e QP) foram resolvidos usando-se, no software livre Scilab®, a implementação do algoritmo de Casas e Pola (1993). Para ilustrar a técnica de alocação de controle baseado em modelo de referência PI foram avaliados os seguintes cenários: (A) manipula-se $F_{1 e}, F_{2 e} \mathrm{e} F$ até o instante de $\mathrm{t}=5 \mathrm{~h}$, quando se realiza uma alteração de setpoint, nessa nova condição ocorre uma falha em que $\mathrm{o}$ atuador em $F_{1 e}$ é travado restando a manipulação de $F_{2 e}$ e $F$, e (B) manipula-se $F_{1 e}, F_{2 e} \mathrm{e} F$ até o instante de $\mathrm{t}=5 \mathrm{~h}$, quando se realiza uma alteração de setpoint, nessa nova condição ocorre uma falha em que o atuador $F_{1 e}$ falha totalmente aberto.
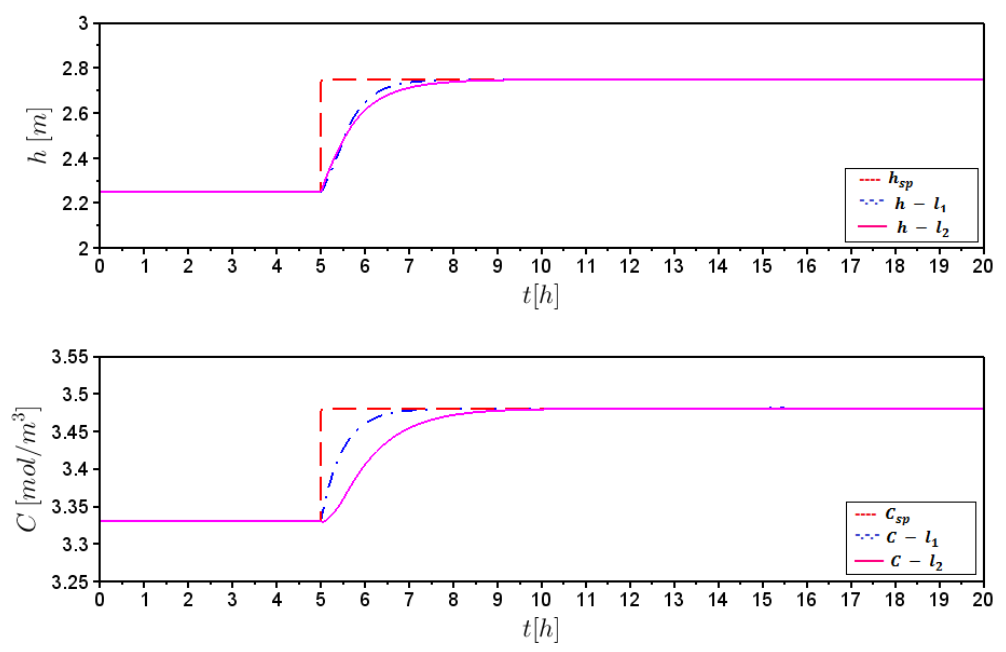

Figura 2 - Comportamento das variáveis controladas - cenário A ( $F_{1 e}$ falha travada).
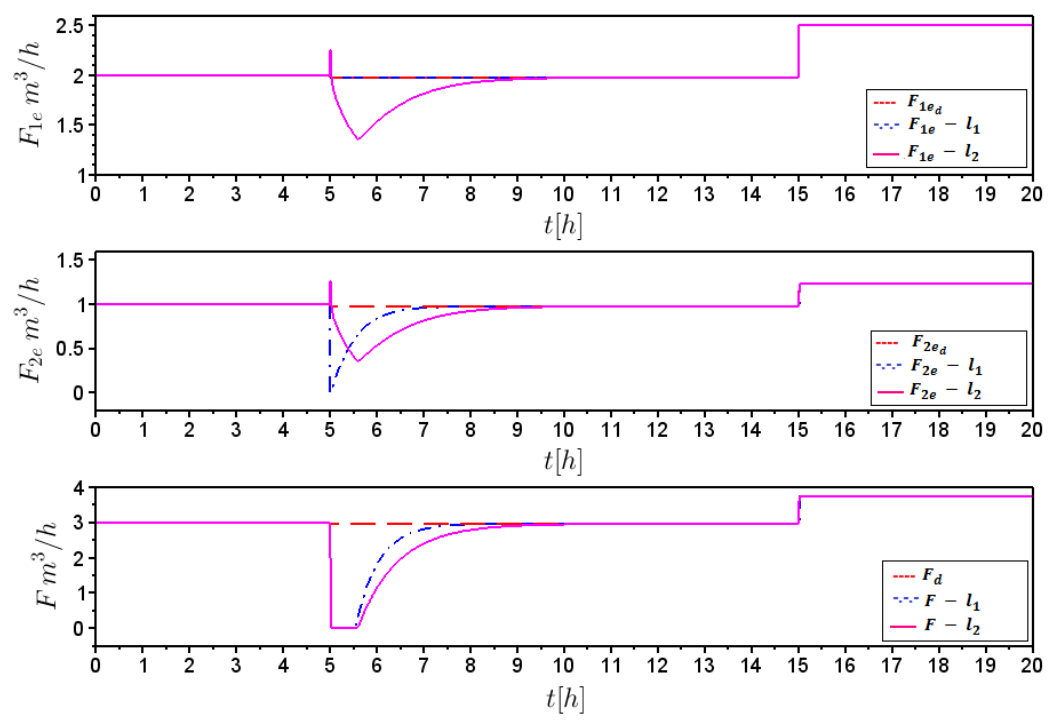

Figura 3 - Comportamento das variáveis manipuladas - cenário $\mathrm{A}\left(F_{1 e}\right.$ falha travada)

No cenário A, com falha o travamento do atuador ocorre com $F_{1 e}=2,4 \mathrm{~m}^{3} / \mathrm{h}$. $\mathrm{O}$ comportamento das variáveis controladas e manipuladas para as alocações com normas $l_{1}$ e $l_{2}$ são mostrados nas Figuras 2 e 3, respectivamente. O comportamento para as variáveis manipuladas, que se estabilizaram atingindo o valor do $u_{d}$, com a alocação com norma $l_{1}$ apresentou comportamento mais rápido para a sintonia utilizada. Pode-se notar que, mesmo com a ocorrência 
da falha, travamento do atuador, o processo manteve-se no valor de referência determinado tanto para o setpoint quanto para o valor desejado para as variáveis manipuladas. Indicando que o procedimento de alocação utilizou o grau de liberdade que restava para controlar perfeitamente o sistema conforme desejado. O esforço de controle foi diferente devido as características dos controladores estudados.

No cenário $\mathrm{B}$, em que o atuador falha totalmente aberto, as Figuras 4 e 5 mostram o comportamento para as variáveis controladas e manipuladas, respectivamente. Neste caso, com a ocorrência da falha, as variáveis controladas tiveram uma pequena variação, próximo ao ponto $t=15 h$ (pouco perceptível na escala do gráfico utilizada), mas logo estabilizando no valor do setpoint. Assim, a técnica de alocação de controle baseado em modelo de referência PI mostrou-se uma ferramenta poderosa para o controle de sistemas com falhas em atuadores, quando graus de liberdade estão disponíveis para alocação do efeito de controle.
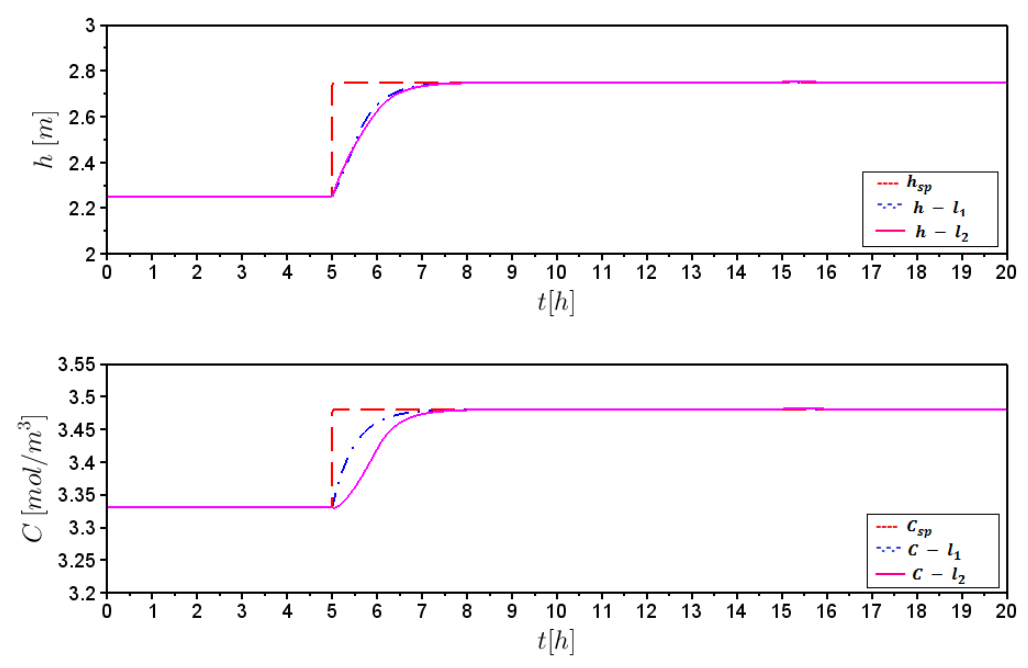

Figura 4 - Comportamento das variáveis controladas - cenário B ( $F_{1 e}$ falha aberta).
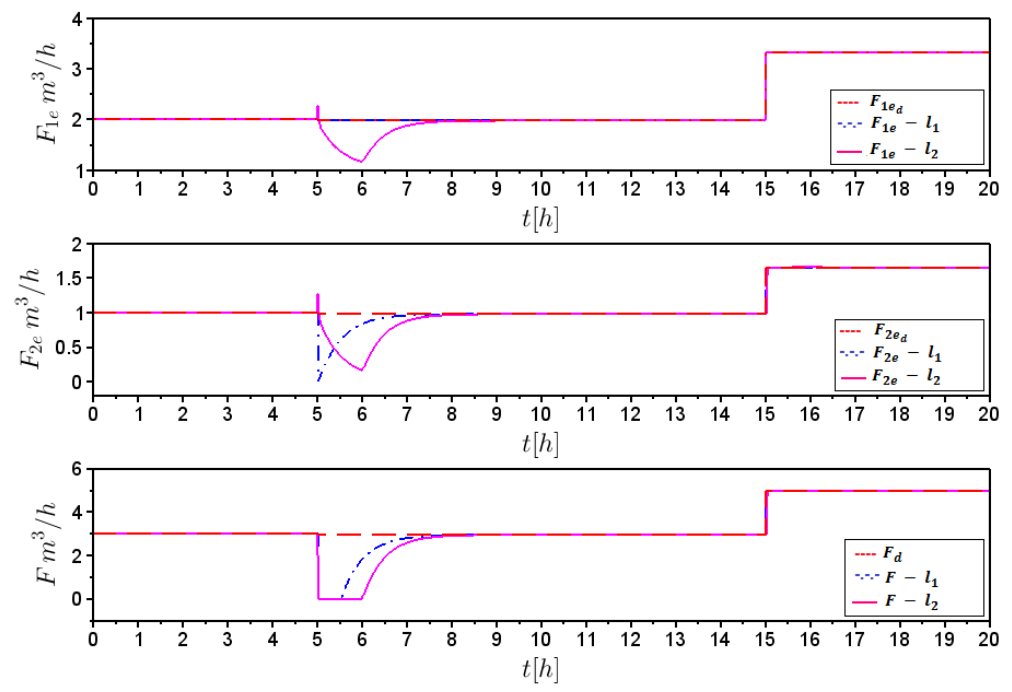

Figura 5 - Comportamento das variáveis manipuladas - cenário $\mathrm{B}$ ( $F_{1 e}$ falha aberta). 


\section{CONCLUSÕES}

As simulações efetuadas para o sistema estudado indicam que a ferramenta de alocação de controle PI pode ser satisfatoriamente implementada, apresentando flexibilidade para tratar de sistemas de controle com falhas em atuadores. A utilização de alocação de controle permite a distribuição dos esforços de controle para as variáveis manipuladas disponíveis (muito útil na presença de falhas para sistemas com mais atuadores que objetivos de controle). A alocação quando o processo de falhas leva a uma perda de graus de liberdade, pode levar a uma região de operabilidade inaceitável, mas, ainda assim, representa um aumento na confiabilidade do processo pois atua como um controlador preditivo de horizonte unitário.

A vantagem da alocação com modelo de referência é a sintonia intuitiva e a possibilidade de projetar o comportamento desejado para a variável controlada. Na utilização das normas $l_{1}$ e $l_{2}$ pode-se notar que a alocação de controle herda as mesmas propriedades conhecidas para controladores preditivos que usem essas normas na defnição do horizonte móvel.

\section{AGRADECIMENTOS}

Os autores agradecem à UFU, à UFTM, à CAPES e à FAPEMIG (Processos PCE00201-14 e PCE-00089-14) pelo apoio concedido.

\section{REFERÊNCIAS}

BASSON, L. Control allocation as part of a fault-tolerant control architecture for UAVs Stellenbosch University - Department Electrical and Electronic Engineering, 2011.

BODSON, M. Evaluation of Optimization Methods for Control Allocation. J. of Guidance, Control, and Dynamics, v. 25, p. 703-711, 2002

BODSON, M.; FROST, S. A. Load Balancing in Control Allocation. J. of Guidance, Control, and Dynamics, v. 34, p. 380-387, 2011.

CASAS, E.; POLA, C. An algorithm for indefinite quadratic programming based on a partial Cholesky factorization, RAIRO-Operations Research, v. 27, p. 401-426, 1993.

HAMAYUN, M.T.; EDWARDS, C.; ALWI, H. A fault tolerant control allocation scheme with output integral sliding modes. Automatica, v. 49, p. 1830-1837, 2013.

KALRA, L.; GEORGAKIS, C; OLIVEIRA-LOPES, L. C. Reference System Model Predictive Control. 1. Continuous Time Formulation and Case Studies on Performance. Ind. Eng. Chem. Res., 41 (13), p. 3199-3212, 2002.

VERMILliON, C.; SUN, J.; BUTTS, K. Predictive Control Allocation for a Thermal Management System Based on an Inner Loop Reference Model - Design, Analysis, and Experimental Results. IEEE Trans. on Control Systems Technology, v. 19, p. 772-781, 2011.

WENDONG, G.; HONGLUN, W. Closed-loop dynamic control allocation for aircraft with multiple actuators. Chinese Journal of Aeronautics, v. 26, p. 676-686. 2013.

ZACCARIAN, L. Dynamic allocation for input redundant control systems. Automatica, v. 45, p. 1431-1438, 2009. 